\title{
OLD AGE IN TURKEY AND WORLD AND SIGNIFICANT PROBLEM OF AGING WORLD: ELDERLY ABUSE AND NEGLIGENCE ${ }^{1}$
}

\begin{abstract}
Developments in he field of medicine and technology in our day, early diagnosis and treatment of diseases' becoming possible thanks to these developments, improvements in standards of living, decrease in fertility rate, extension of expected life time result in aging of World population. Aging world population brings problems on old age into agenda. One of these problems is elderly abuse and negligence.

Being a new subject matter for our country which was previously seen as a taboo throughout he world and started being paid necessary attention nowadays, elderly abuse is called as "all kinds of violent conducts directed to elders by relatives or other caregivers".

This study aims at assessing the situation in Turkey and world on the issue of elderly abuse and negligence and to set forth a projection concerning old age on the basis of demographic data in Turkey.
\end{abstract}

Key Words: Old age, elderly abuse, elderly negligence

\section{INTRODUCTION}

Elderliness is a period in which dependency increases due to the decrease in both physical and cognitive functions of the individual, income level, dignity, status and support of those around him decrease (Akdemir et al., 2008; Gülen et al., 2013).Old age is a universal process in every living creature, which cannot be avoided, causes a decrease in all functions and brings biological, chronological and social problems together (Uysal, 2002; Sayan et al.,2004; Yeşil et al., 2016).

The World Health Organization defines old age as "diminished or largely lost ability to adapt to environmental factors", and adopts age 65 as the onset of old age, age 80 and older as the advanced age of old age (Gülen et al.,2013; Molinelli et al., 2017).Today, the developments in the field of medicine and technology, the early diagnosis and treatment of diseases made possible, the presence of individuals who are more conscious about healthy living, the improvement of living standards, decreased fertility, increased life expectancy and increased the proportion of elderly population (Sayan et al.,2004; Arpac1 et al., 2017; Uysal, 2002; Yeşil et al., 2016; Kıssal et al., 2009; Cornell et al., 1982).

According to the World Population Estimate Report, which was prepared by the United Nations in 2017, "the world population in 2030 8.6, 2050 9.8, 210011.2 billion will exceed; currently 962 million in the world of 60 years and over, 3 times as much as 2100 to 3,1 billion is expected to increase" is expressed (https://aa.com.tr/tr/dunya/bmnin-2050-dunya-nufusu-tahmini-98-

\footnotetext{
${ }^{1}$ This study was presented as a seminar at Selcuk University, Institute of Health Sciences, Department of Health Management.

${ }^{2}$ Selcuk University, Faculty of Health Sciences, Department of Health Management. melekyagci@selcuk.edu.tr, ORCID ID: https://orcid.org/000-0003-0314-247X
} 
milyar/846660, 27.04.2018). As pointed out in the World Population Estimation Report, the world population is aging and the increase in the number of aging populations raises issues related to old age (Uysal, 2002). In this context, it is necessary to emphasize the elderly abuse and neglect which has become one of the issues that should be given priority in our country as well as the elderly population in our country (Lök, 2015; Akdemir et al.,2008).

\section{Elderly Abuse And Negligence}

The elderly/old age abuse, which is considered as a very recent issue in our country, is defined as "any violent behavior directed by relatives of the elderly or other caregivers" (Daşbaş, 2014). As can be understood from the definition, elderly abuse is a form of violence and it has started to emerge as a result of domestic violence studies conducted in the field of child abuse with women (Artan, 2016,).

Elder abuse and neglect in the literature were first used by Baker and Burston in 1975 by the term" granny battering" (Kissal et al.,2009; Artan, 2016). The abuse and neglect of the elderly began to be treated as an important old age problem apart from domestic violence as conducted within the studies (Gökçe, 2017).

After the concept emerged, legal regulations were introduced in the United States of America, and in 1976, the obligation to make a legal declaration on elder abuse was adopted in the United States with 43 provinces, Canada and Israel (Artan, 2016; Daşbaş, 2014). In 1990, the prevalence study was accepted as the first data for elderly abuse in the UK (Perel-Levin, 2008). Attention to elder abuse has led to the establishment of the international network for the Prevention of elder abuse (INPEA) in 1997 around the world. "The Toronto Declaration on Global Prevention of Elder Abuse", adopted by the World Health Organization in 2002, aims to prevent elder abuse and emphasizes the importance of participation of many segments of society in the Prevention of elder abuse (Carmona et al.,2018). In the Toronto Declaration, "there is no single or recurrent inappropriate action or appropriate action that harm or stress the elderly in any relationship with a trust expectation" (World Health Organization, 2002).

In our country, there is no regulation to prevent elder abuse and neglect yet, and only reports on the issue are evaluated by Alo 183 line, which is connected to the Ministry of Family, Social Labour and Social Services (Artan, 2016).

In general, it is possible to define the abuse of the elderly as any physical, psychological or economic behavior or neglect that threatens or damages the health or well-being of the elderly (Akdemir et al.,2008).

Elder abuse has become a social problem in many parts of the world, which has recently been recognized as a private matter that should be kept unnoticed until recently (Carmona et al.,2018).

The fact that World Health Organization (WHO) data shows that 6 out of 6 elderly people in the world are being exploited reveals the importance of the problem (World Health Organization, 2018).

In order to give importance to the problem of elderly abuse, it is necessary to define the problem firstly (Comijs et al.,1998). In this context, in order to define elder abuse, it will first be classified into three groups as family, institutional and self-neglect, and then it will be referred to the types of age abuse, and it will be possible to get a better understanding of the issue.

Family abuse is the abuse of older individuals by family members in their homes or in the homes of other individuals who take care of them (Akdemir et al., 2008). According to World Health Organization data, $90 \%$ of the abusers are composed of family members. These abusers are mostly adults and spouses (World Health Organization, 2018). 
Elder abuse can occur not only in the home environment of elderly people but also in housing and hospital settings. Such abuse is considered to be corporate abuse (Molinelli et al.,2017).

Self-neglect is that the elderly do not show the care and attention necessary for themselves (Fadılıoğlu et al.,2012).

As noted in earlier sequences, elder abuse is classified into three groups: familial, institutional and self-neglect. Following this classification, the types of abuse against the elderly will be mentioned.It has been observed that the types of abuse in the literature about elder abuse are usually examined in five headings as physical abuse, sexual abuse, psychological abuse, financial abuse and neglect (Kleinschmidt, 1997; Lachs et al.,2015). The definitions of the above mentioned types of abuse are given below:

Physical abuse: Violence in the ways such as physically hitting the elderly, slapping, causing the elderly to bodily injury, etc. is considered physical abuse (Levine, 2003)

Sexual Abuse:Sexual abuse, which can also be categorized into physical abuse (Choi et al.,2000) is a close contact with the old person without his / her consent (Kleinschmidt, 1997). Older individuals who have been sexually abused, embarrassed, think that people who take care of themselves will be punished, etc. sexual abuse mostly been keeping reasons (Gökçe, 2017; Akdemir et al.,2008). Sexual abuse is usually less reported and less reported, and probably less recorded, for reasons such as these and similar reasons (Molinelli et al.,2017).

Psychological/ Emotional Abuse: The behavior of the elderly person to feel bad from the psychological aspect is considered as psychological abuse. In this context, such as insult, reprimand, intimidation, intimidation, and contempt of the elderly person are types of psychological abuse (Gökçe, 2017).

Economic Abuse:It includes illegal misuse or theft of the financial resources, property or assets owned by the old (Levine, 2003; Choi et al.,2000). As in other types of abuse in economic abuse, individuals are trusted by the elderly (Fadılıoğlu et al., 2012).

Signs of economic abuse of the elderly, irregular withdrawals from the bank accounts of the elderly person, decreases in assets, the jewelery and jewelry of the elderly individual such as the disappearance of valuables, etc. Shows (Artan, 2016).

Neglect: In case of violations of human rights, legal and medical rights, the right to take decisions and decisions about the elderly person by the institutions responsible for the care of the individuals or the elderly person arises (Gökçe, 2017).

Among the negligence towards the elderly person, it is exemplary that individuals who undertake care of the needs of elderly people (eating, drinking, dressing, cleaning, etc.) are abstaining from being reluctant to take the responsibility of the elderly person and leaving the elderly person both physically and emotionally (Gökçe, 2017).

In 52 studies in 28 countries, including 12 low- and middle-income countries, supported by the World Health Organization (WHO) and published at Lancet Global Health; Almost $16 \%$ of individuals aged 60 and over were exposed to abuse. $11,6 \%$ of these individuals were reported to be emotional, 6,8\% financial, 4,2\% neglect, 2,6\% physical, and 0,9\% sexual abuse (World Health Organization, 2018).

\subsection{Risk Factors Causing Elder Abuse and Neglect}

There are a number of risk factors that can lead to elder abuse and neglect, which are considered as one of the major problems that have come to the agenda with the rapid rise of the aging population. These factors can be evaluated as the reasons for giving care, cultural, institutional and elderly individuals (Akdemir et al.,2008).

Elderly individuals who are dependent on others in order to meet the most important risk group especially in elderly abuse and negligence, who live alone, have a number of health problems 
(American Psycholigical Association, 2012; Yeşil et al.,2016). In addition, the physical and mental insufficiency of the elderly person, the presence of alcohol or drug addiction, the presence of verbal behaviors with violence, abuse and neglect are among the factors related to the elderly individual (Akdemir et al.,2008).

As for the elderly person living with his family, the family history of violence, the different lifestyles resulting from the differences between generations, and the fact that the elderly create an economic burden for the family constitute the familial factors (American Psycholigical Association, 2012).

Elder abuse and neglect can be caused by factors related to the elderly individual and his family, and may cause factors related to caregiver taking care of the elderly individual. Factors related to caregivers may include the presence of physical and psychological illnesses in the caregiver, being addicted to alcohol or drugs, being addicted to violence, and being financially dependent on the elderly individual (American Psycholigical Association, 2012; Gökçe, 2017).

A variety of cultural attitudes and behaviors can also be caused by elderly abuse and neglect. The most important reason for the abuse of cultural attitudes and behavior is the lack of respect for the elderly person. The lack of importance to the elderly is also an important cause of cultural factors (American Psycholigical Association, 2012).

Among the institutional factors, the individuals who take care of elderly people living in institutions are employed for long hours with low wages, the managers and other employees are insensitive to elder abuse and neglect, etc (Akdemir et al.,2008; Gökçe, 2017).

\subsection{Determination and Prevention of Elder Abuse and Neglect}

A multidisciplinary approach is important in determining the elderly abuse and neglect. Here, people who are witnessing the abuse of the elderly person, especially the elderly person in the health sector, physicians, nurses and other health professionals, and social workers play a key role in determining abuse and neglect (Sayan et al.,2004; Choi et al., 2000).

Health professionals are especially important in determining the elderly abuse. The process begins with the suspicion of the elderly person in determining the elderly abuse and neglect. In the event of any doubt, health personnel should take laboratory tests from the elderly person, perform a full physical examination including sexual organs, fracture of the elderly person's body, etc. X-ray studies, such as X-ray, magnetic resonance, should be used when suspected (Levine, 2003). In addition to physical examination, health professionals should be able to observe whether the elderly person has social problems (Molinelli et al.,2017). Where it is believed that the elderly have been abused, health professionals must take on the role of rescuers who can extend the hand of aid to the victims and guide them to the authorities.

However, health personnel alone are not sufficient to prevent elder abuse and neglect, public health, social, medical and legal activities should be identified (World Health Organization, 2008). Elder abuse and neglect will continue to be ignored and remain as a taboo unless there is an interdisciplinary response to this kind of abuse and neglect (Perel-Levin, 2008).

The first step in the prevention of the abuse and neglect of the elderly is to be aware of the fact that no one should be subjected to any insulting or negligent behavior, including violence and profanity (American Psycholigical Association, 2012). In having such a consciousness, especially families and educational institutions have great responsibilities.Following such a social step, the training to be given to people about the abuse of the elderly is an important building block for the prevention of elderly abuse and neglect.In particular, health professionals who care for the elderly should be trained (Akdemir et al.,2008; Kissal et al., 2009). Providing social support to caregivers of elderly individuals is one of the factors that can prevent abuse and neglect. 


\section{METHOD}

This study aims to present a projection based on data from secondary sources of old age and abuse in Turkey and in the world, and to assess the situation in Turkey and in the world on elder abuse and neglect.

\section{RESULTS}

\subsection{Aging Results}

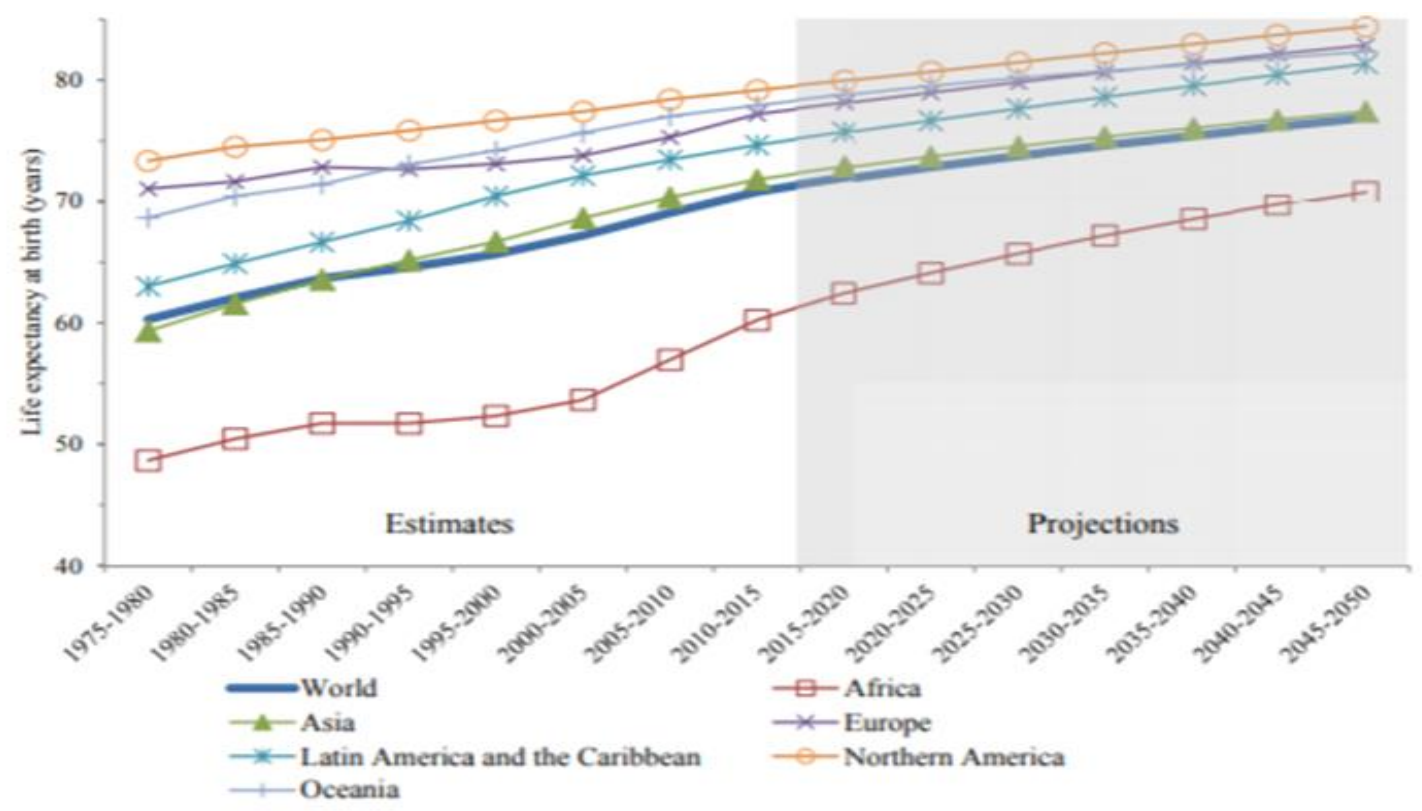

Figure 1: Life Expectancy at Birth, World

Source: World Health Organization, 2018.

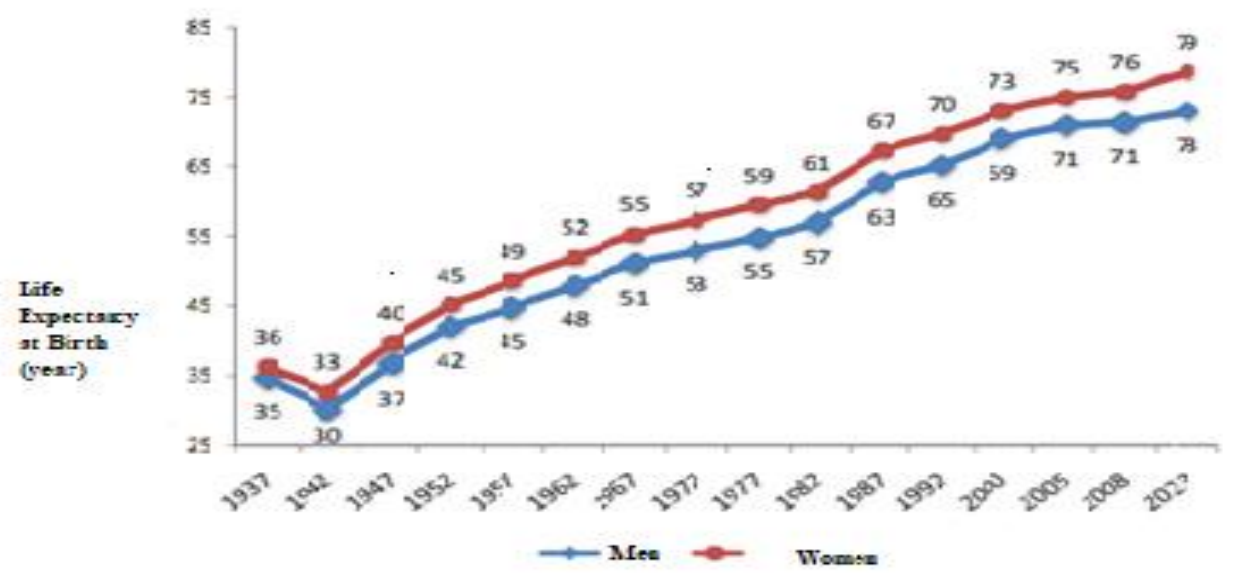

Figure 2: Changes in Expectation of Life Expectation at Birth, Turkey

Source: Turkey Demographic Transformation, 2008. 
Figure 1 and Figure 2 show the expected life expectancy at birth, which is one of the important factors in the aging of the world population. As we can see, life expectancy in both the world and in our country has increased over the years, creating one of the building blocks of aging.

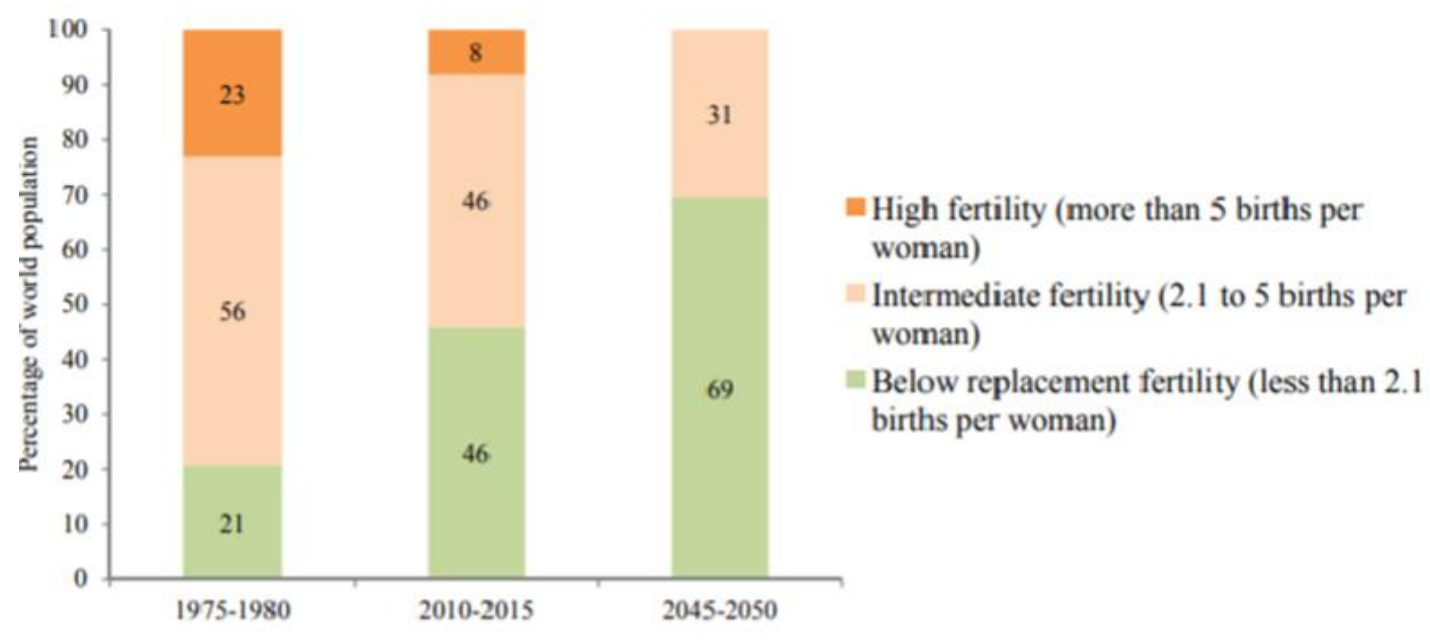

Figure 3:Distribution Of The World Population By Total Fertility Level

Source: World Population Prospects, 2017.

Figure 3 and Figure 4 also show changes in fertility levels, which are considered to be one of the important indicators of aging in the world. When examined, there are expectations that high fertility rates will be eliminated in the world between 2045-2050 and low fertility rates will be increased.

Figure 4 shows the rate of increase in the population of Turkey. Although population growth rate is declining, the population continues to increase is considered to be an indicator of low fertility and older population growth.

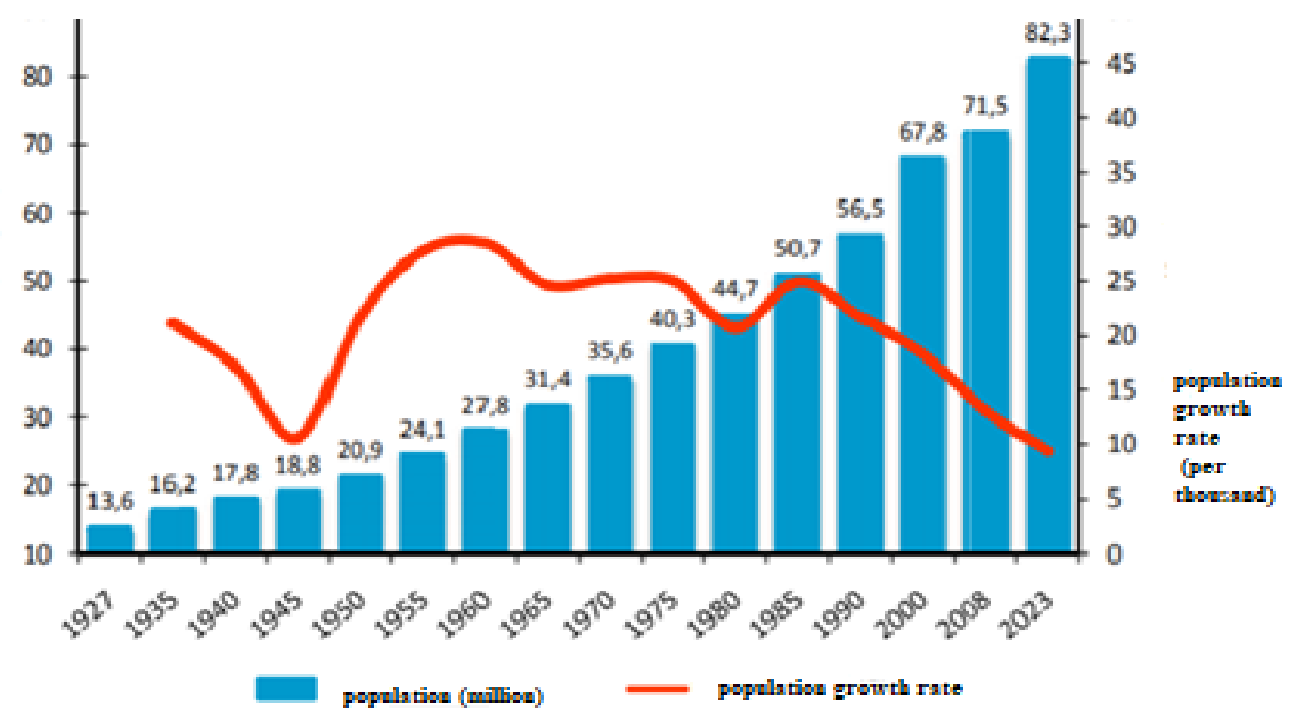

Figure 4: Population Size and Changes İn The Rate Of Growth, turkey Source: Demographic Transformation Of Turkey, 2008. 
In the above paragraphs, a number of indicators from both the world and Turkey indicate that the world population is aging. According to the world population forecast report prepared by the United Nations in 2017, "the world population in 2030, 8.6, 2050, 9.8, 2100, 11.2 billion will exceed; around the world 962 million 60 years and above, the population is expected to increase by 3 times to 3.1 billion by 2100," it is stated (https://aa.com.tr/tr/dunya/bmnin-2050-dunyanufusu-tahmini-98-milyar/846660, 27.04.2018).

\begin{tabular}{lrrrr}
\hline & Region & \multicolumn{4}{c}{ Population (millions) } \\
\cline { 2 - 5 } & 7550 & 2030 & 2050 & 2100 \\
\hline World & 1256 & 1704 & 2528 & 4468 \\
Africa & 4504 & 4947 & 5257 & 4780 \\
Asia & 742 & 739 & 716 & 653 \\
Europe & 646 & 718 & 780 & 712 \\
Latin America and the Caribbean $\ldots \ldots \ldots \ldots . .$. & 361 & 395 & 435 & 499 \\
Northern America & 41 & 48 & 57 & 72 \\
\hline
\end{tabular}

Figure 4: World Population

Source: World Population Prospects, 2017.

As indicated in the World Population prediction report, the world population is aging and the increase in the number of aging population raises issues related to old age (Uysal, 2002)..

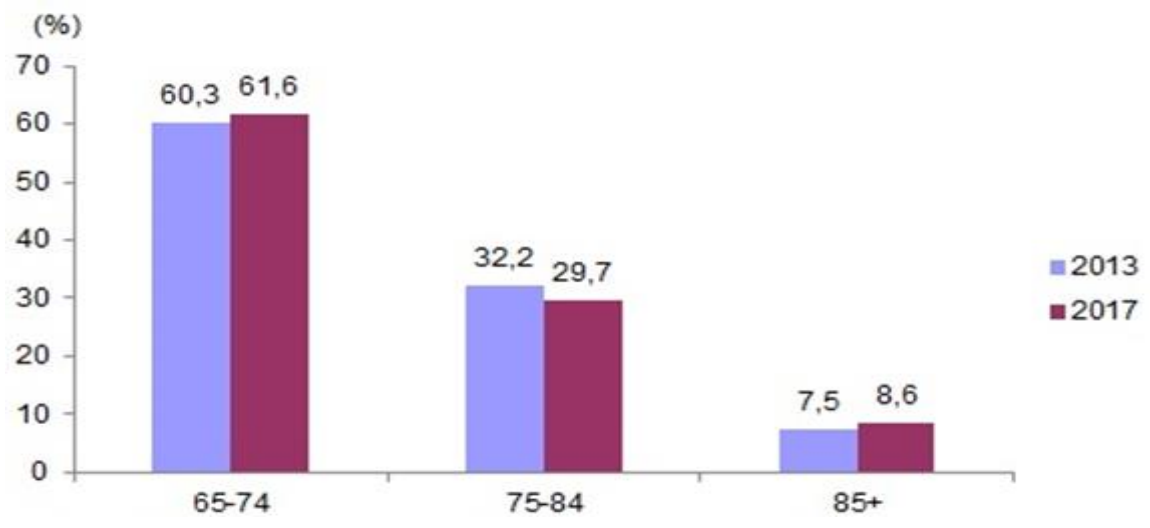

Figure 5: Elderly Population, Turkey

Source: Demographic Transformation Of Turkey, 2008.

In this context, as can be seen in Figure 5, the elderly population is increasing in our country as in the world. Elderly abuse and neglect, which has become one of the issues that needs to be prioritised along with the growing elderly population, needs to be emphasized (Lök, 2015; Akdemir et al.,2008). .

\subsection{Findings of elder abuse and neglect}

According to World Health Organization (WHO) data, the World Health Organization (who) shows that 1 out of 6 of the aged population around the world are being exploited (World Health Organization, 2018) shows that elder abuse and neglect are an important problem.

Again, the World Health Organization's Global Situation Report on Violence Prevention (2014) in 133 countries, which is supported by high, medium and low income, provides a striking conclusion that the elderly are most abused in the highest-income countries (Figure 6). 


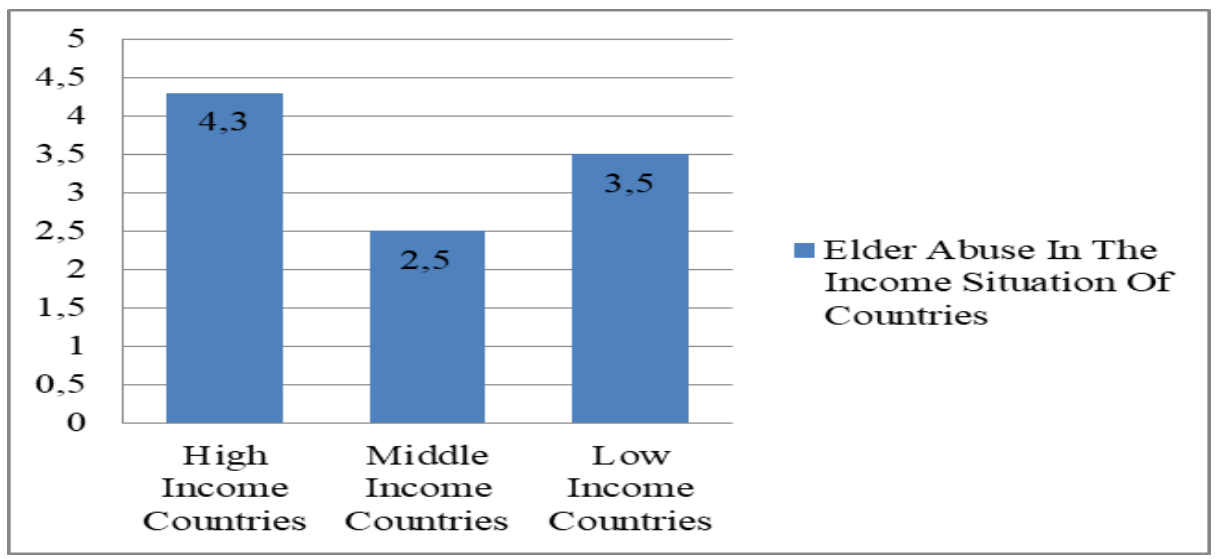

Figure 6: Elder Abuse In The Income Situation Of Countries

Source: Global Status Report On Violence Prevention, 2014.

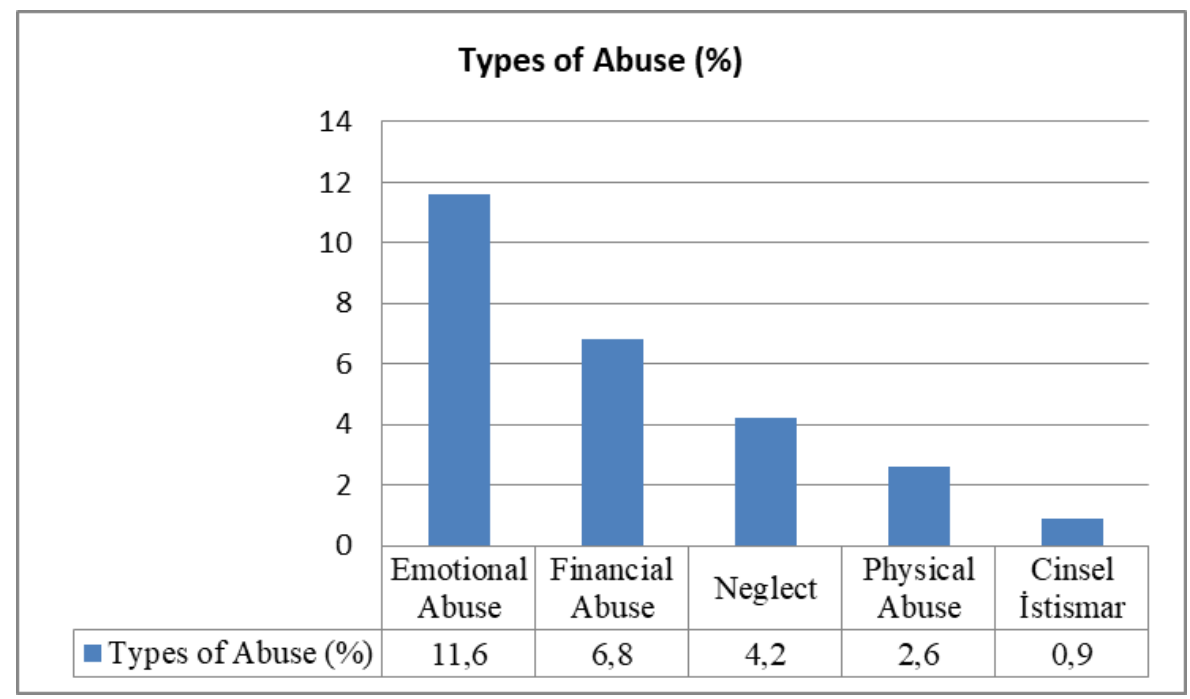

Figure 7: Types Of Elder Abuse

Source: World Health Organization, 2018.

In a study conducted in 28 countries, including 12 low-income and middle-income countries, supported by the World Health Organization (who), Lancet Global Healthcare found that almost $16 \%$ of adults aged 60 and over were abused. $11.6 \%$ of these individuals were emotional, $6.8 \%$ were financial, $4.2 \%$ were neglect, $2.6 \%$ were physical, and $0.9 \%$ were subjected to sexual abuse (World Health Organization, 2018).

\section{RESULT}

Today, social, economic, technological and medical developments in the field of medicine and living standards, along with the expected life expectancy of individuals has extended(Sayan et al.,2004; Arpacı et al., 2017; Uysal, 2002; Yeşil et al., 2016; Kıssal et al., 2009; Cornell et al., 1982). According to the United Nations World Population prediction report, the world population is rapidly aging https://aa.com.tr/tr/dunya/bmnin-2050-dunya-nufusu-tahmini-98-milyar/846660 27.04.2018), and the increase in the number of elderly population raises many problems related to old age. One of these problems is the abuse and neglect of the elderly, which is defined as "any violent behaviour directed by relatives or caregivers of the elderly"(Daşbaş, 2014). 
According to data from the World Health Organization, 6 out of the aged people in the world are being abused (World Health Organization, 2018). 11.6\% of these individuals were subjected to emotional, $6.8 \%$ to financial, $4.2 \%$ to neglect, $2.6 \%$ to physical, and $0.9 \%$ to sexual abuse (World Health Organization, 2018).

Although there are important tasks for health workers in the prevention of elder abuse and neglect, which is emphasized as an important problem with the aging world and whose importance has been understood, there is a multidisciplinary approach that is not sufficient for health workers alone (Choi et al.,2000; Perel-Levin, 2008). However, in the prevention of elder abuse and neglect, giving education to family members who care for the elderly, staff and health professionals who work in institutions that care for the elderly, and social support to family members who care for the elderly are an important step in solving the problem (Akdemir et al.,2008; Kissal et al., 2009).

Despite the fact that there is a worldwide awareness of old abuse and neglect, which was seen as taboo in the past with the aging world population, there is no step taken on this issue in our country. In the absence of such a step in our country, it is thought that the importance and respect given to an elderly individual is effective in Turkish culture. However, the rapidly changing world and especially the woman, who is an important member of the family, has difficulty in providing care to the elderly person with the proliferation of core family structures, and the choice of elderly care homes, as well as the issue of abuse against the elderly is thought to come to the agenda for our country. However, considering the aging population of our country, making legal arrangements for elder abuse and neglect for our country is an important step in ensuring awareness of the issue and preventing serious problems in the future.

\section{REFERENCES}

Akdemir N., Görgülü Ü. \& Çınar F. İ. (2008). Elderly Abuse and Neglect. Faculty of Health Sciences Nursing Journal,68-75.

Molinelli A., Ventura F., Pinto S.L., Drommi M. \&Stefano F.D. (2017). Elder abuse in Europe's "most elderly" city: an assessment of the phenomenon and an analysis of the data from the Penal Court of Genoa from 2010 to 2015. Aging Clin Exp Res, 1285-1290.

American Psycholigical Association (2012). Elder Abuse\&Neglect In Search Of Solutions.

Arpacı F. \&Bakır B. (2017). Elderly Abuse and Neglect. Turkısh Journal of Social Research, 691703.

Artan T. (2016). Financial Abuse as a Type of Elderly Abuse among Elderly People Residing in Senior Centers. HSP, 3(1), 48-56.

Carmona J. M., López P. J., Coimbra A. I., Gálvez R. M., Goergen T.\& Rodríguez A. (2018). Elder Abuse in a Developing Area in Bolivia. Journal of Interpersonal Violence, 33(2), 339-356.

Choi, N.G. \& Mayer J. (2000). Elder Abuse, Neglect, and Exploitation: Risk Factors and Prevention Strategies. Journal of Gerontological Social Work, 33(2), 5-25.

Comijs H.C., Pot A.M., Smit J.H., Bouter L.M. \&CeesJonker. (1998). Elder Abuse in the Community: Prevalence and Consequences. Jags, 885-888.

Cornell C.P. \& Gelles R.J. (1982). Elder Abuse: The Status of Current Knowledge. Family Relations, 31(3), 457-465.

Daşbaş S. (2014). doctoral thesis. Reviews of people who are 65 and older about domestic elder abuse. Ankara: Hacettepe University Institute of Social Sciences.

Fadılıoglu Ç.\& Şenuzun A. (2012). Approach to abuse and neglect in the elderly. Ege Journal of Medicine, 69-77. 
Gökçe N. (2017). Elder Abuse and Neglect. Health Academy Kastamonu,2(2), 100-111.

Gülen M., Aktürk A., Acehan S., Seğmen M.S., Açıkalın A.\& Bilen A. (2013). Elder Abuse and Neglect. Archives Medical Review Journal, 22(3), 393-407.

Hacettepe University Institute of Population Studies (2008). Turkey Demographic Transformation. Ankara.

Kıssal A.\&Beşer A. (2009). Identifying and Evaluating Elder Abuse and Neglect. TAF Preventive Medicine Bulletin, 8(4), 357-364.

Kleinschmidt K.C. (1997). Elder Abuse: A Review. ANNALS OF EMERGENCY MEDICINE, 30(4), 463-472.

Lachs M.S. \&Pillemer K.A. (2015). Elder Abuse. The New England Journal of Medicine, 19471956.

Levine J.M. (2003). Elder neglect and abuse: A primer for primary care physicians. Geriatrics, 58(10), 37-44.

Lök N. (2015). Elder Abuse and Neglect in Turkey: A Systematic Review. -Current Approaches in Psychiatry, 7(2), 149-156.

Perel-Levin S. (2008). Discussing Screening for Elder Abuse at Primary Health Care level. Switzerland: WHO.

Sayan A.\& Durat G. (2004). Elder Abuse and Neglect: Preventive Intervention. Journal of Ataturk University School of Nursing, 7(3), 97-106.

Uysal A. (2002). A Wor/d-Wide Problem: Elder Abuse and Neglect.Family and Society, 2(2).

World Health Organization (2002). The Toronto Declaratıon on the Global Prevention of Elder Abuse, Switzerland.

World Health Organization (2008). A Global Response to Elder Abuse and Neglect: Building Primary Health Care Capacity to Deal with the Problem Worldwide: Main Report.

World Health Organization (2014). Global Status Report On Violence Prevention 2014, Switzerland.

World Population Prospects (2017). New York: United Nations.

Yeşil P., Taşc1 S. \& Öztunç G. (2016). Elder Abuse and Neglect. Duzce University Journal of the Institute of Health Sciences, 6(2), 128-134. 